\title{
FITOSSOCIOLOGIA DOS ESTRATOS ARBÓREO E DE REGENERAÇÃO NATURAL EM UM POVOAMENTO DE ACÁCIA-NEGRA (Acacia mearnsii De Wild.) NA REGIÃO DA FLORESTA ESTACIONAL SEMIDECIDUAL DO RIO GRANDE DO SUL
}

\author{
PHYTOSOCIOLOGY OF THE ARBOREAL AND NATURAL REGENERATION STRATA IN A \\ BLACK-WATTLE (Acacia mearnsii De Wild.) PLANTATION IN THE REGION OF SEMIDECIDUIS \\ SEASONAL FORESTS OF RIO GRANDE DO SUL
}

\author{
Silas Mochiutti ${ }^{1}$ Antonio R. Higa ${ }^{2}$ Augusto A. Simon ${ }^{3}$ \\ RESUMO
}

O presente estudo analisa a composição e a estrutura da regeneração de espécies nativas arbóreas em um povoamento de acácia-negra, estabelecido há 16 anos em área ripária, e verifica a possibilidade da acácia-negra constituir-se numa invasora desse ambiente. $O$ estrato arbóreo (circunferência a 1,3 m de altura $(\mathrm{CAP}) \geq 15 \mathrm{~cm}$ ) foi avaliado em 12 parcelas de $100 \mathrm{~m}^{2}$, alocadas em quatro blocos perpendiculares a maior pendente da área. $\mathrm{O}$ estrato de regeneração natural $(0,3 \mathrm{~m}$ de altura a $\mathrm{CAP}<15 \mathrm{~cm})$ foi amostrado em duas subparcelas de $9 \mathrm{~m}^{2}$, demarcadas em vértices opostos de cada parcela. O estrato arbóreo apresentou 26 espécies de 14 famílias e o estrato de regeneração natural 49 espécies de 23 famílias. O índice de diversidade de Shannon para espécie, considerando as parcelas como um todo, foi de 2,60 e 3,06 para os estratos arbóreo e de regeneração natural respectivamente. Entre as espécies nativas, Casearia sylvestris, Myrsine lorentziana e Zanthoxylum petiolare apresentaram o maior valor de importância no estrato arbóreo e Faramea marginata, Myrsine lorentziana e Myrcia glabra a maior densidade no estrato de regeneração natural. As características ecológicas das espécies encontradas nos diversos estratos de altura indicaram que o processo de sucessão florestal está em evolução. A acácia-negra não se regenerou na área e as árvores plantadas estão em senescência, sendo encontradas somente cem plantas/ha, que representa apenas $4,5 \%$ da população original. Dessa forma, a acácia-negra não se constitui numa possível invasora desse ambiente.

Palavras-chaves: planta invasora; regeneração natural; estrutura; composição florística.

\section{ABSTRACT}

This study analyzes the structure and floristic composition of the regeneration of native forest species under black-wattle plantation, established 16 years ago in a riparian area, and it verifies the possibility of the black-wattle be considered an invasive species of this environment. The tree stratum (circumference at breast height $(\mathrm{CBH}) \geq 15 \mathrm{~cm}$ ) was evaluated in 12 plots of $100 \mathrm{~m}^{2}$, established in four blocks. The natural regeneration stratum $(0.3 \mathrm{~m}$ height to $<15 \mathrm{~cm} \mathrm{CBH})$ was evaluated in two subplots of 9 $\mathrm{m}^{2}$, established in opposite vertexes of each plot. The tree stratum was composed by 26 species of 14 families and the natural regeneration stratum by 49 species of 23 families. The Shannon diversity index for species, considering all plots, was 2.60 and 3.06 to the tree and natural regeneration strata, respectively. The native species, Casearia sylvestris, Myrsine lorentziana and Zanthoxylum petiolare presented the larger importance value in the tree stratum and Faramea marginata, Myrsine lorentziana and Myrcia glabra the biggest density in the natural regeneration stratum. The ecological characteristics of the species found in several height strata indicated that the forest succession process is in evolution. The black-wattle did not regenerate in this area and the planted trees of this species are in the senescence phase. Only 100 tree/ha of black-wattle were found, which represents $4.5 \%$ of the original population. Thus, black-wattle is not an invasive species for this environment.

Keywords: invasive plant; regeneration; structure; floristic composition.

1. Engenheiro Agrônomo, Dr., Pesquisador da Embrapa Amapá, Rodovia Juscelino Kubitschek, km 5, Caixa Postal 10, CEP 68906-970, Macapá (AP). silasmochiutti@cpafap.embrapa.br

2. Engenheiro Florestal, PhD., Professor do Departamento de Ciências Florestais, Escola de Florestas, Universidade Federal do Paraná, Rua Lothário Meissner, 3400, Jardim Botânico, Campus III, CEP 80210-170, Curitiba (PR). higa@ufpr.br

3. Engenheiro Florestal, Técnico da TANAGRO, Empresa do Grupo TANAC S.A., Rua Torbjorn Weibull, 199, CEP 95780-000, Montenegro (RS) aasimon@tanac.com.br

Recebido para publicação em 5/06/2006 e aceito em 22/11/2007. 


\section{INTRODUÇÃO}

A acácia-negra (Acacia mearnsii De Wild., Fabaceae) é cultivada, especialmente por pequenos produtores, em cerca de cinqüenta municípios do Rio Grande do Sul, alcançando em 2006 uma área plantada de 152 mil hectares (ABRAF, 2007). Originária da Austrália e da Tasmânia, é uma árvore pioneira de rápido crescimento e vida curta (SHERRY, 1971), eficiente fixadora de nitrogênio (AUER e SILVA, 1992) e indicada para recuperação de áreas degradadas (KANNEGIESSER, 1990).

Para atender à legislação sobre a restauração da cobertura vegetal nativa em áreas de preservação permanente (APPs) que tiveram essa vegetação suprimida, plantações de acácia-negra localizadas dentro de APPs estão sendo abandonadas em pé ou após a realização da colheita florestal. Nos dois casos, essas áreas são mantidas sem intervenções para a recuperação da cobertura vegetal nativa. Nessas áreas, a capacidade de estabelecimento de uma regeneração natural é um indicador da evolução da APP para oferecer os serviços ambientais de proteção dos mananciais e restabelecimento da biodiversidade original, bem como contribuir para alcançar a pretendida sustentabilidade.

Plantações florestais podem apresentar as mesmas funções das florestas secundárias no restabelecimento da riqueza de espécies florestal de áreas degradadas (LUGO, 1997), sendo considerada uma opção viável para acelerar o processo de recuperação e restauração da produtividade, biodiversidade e outros serviços ambientais dessas áreas (PARROTTA et al., 1997a).

Povoamentos florestais monoespecíficos de Eucalyptus (CALEGARIO et al., 1993; SILVA Jr. et al., 1995; NERI et al., 2005), Pinus (LOMBARDI e MOTTA Jr, 1992; ANDRAE et al., 2005) e Tectona grandis L. f. (HEALEY e GARA, 2003) apresentaram uma elevada regeneração natural, com apreciável diversidade de espécies florestais nativas.

Leguminosas arbóreas podem funcionar como facilitadoras da sucessão natural em áreas degradadas, pela capacidade dessas espécies em melhorar as condições dos sítios. Campello (1999) comparou a regeneração natural em plantios de leguminosas nativa (Sclerolobium paniculatum Vogel) e exótica (Acacia mangium Willd.), e de não-leguminosas nativa (Goupia glabra Aubl.) e exóticas (Eucalyptus citriodora Hook. e Eucalyptus pellita F. Muell.) em área degradada pela remoção total do horizonte A (área de empréstimo). Esse autor (ibidem) concluiu que a regeneração natural das espécies nativas foi beneficiada pelo plantio de leguminosas arbóreas, independentemente de ser nativa ou exótica.

A regeneração de espécies arbóreas nativas nas plantações florestais está condicionada à oferta de propágulos e a capacidade desses em se estabelecerem e se desenvolverem nas condições ambientais do sítio. A oferta de propágulos está em função da distância e da qualidade da fonte e dos agentes dispersores. Em área de restauração florestal em Trombetas, PA, Parrotta et al. (1997b) concluiram que quanto maior a distância da floresta primária menor foi a abundância e diversidade de espécies.

A composição e estrutura florística da regeneração de espécies nativas em um sub-bosque de Eucalyptus saligna $\mathrm{Sm}$. dependeu das características edáficas e da proximidade de fontes de propágulos (SARTORI et al., 2002). NAPPO et al. (2000) encontraram influências significativas das variáveis edáficas sobre a densidade e diversidade da regeneração natural em plantio de Mimosa scabrella Benth. implantado para reabilitação de uma área minerada.

A qualidade da fonte de propágulos refere-se à riqueza de espécies da vegetação circunvizinha. A diversidade das espécies nativas encontradas na regeneração de povoamentos florestais normalmente é menor que a encontrada na vegetação nativa próxima (HEALEY e GARA, 2003; SAPORETTI Jr. et al., 2003; NERI et al., 2005), porém esses povoamentos também podem receber propágulos de fontes mais distantes (CHADA et al., 2004). Em regiões tropicais, a disseminação de sementes por animais é a forma predominante de dispersão de propágulos, tendo essa síndrome um papel fundamental na diversidade florestal de áreas recolonizadas (WUNDERLE Jr., 1997).

Por outro lado, algumas espécies florestais exóticas podem se tornar invasoras de ambientes, mantendo uma população de plantas na área da plantação, pelo recrutamento de novos indivíduos (RICHARDSON et al., 2000), e impedir ou retardar a regeneração natural das espécies nativas (REIS et al., 1999). Nesse sentido, a acácia-negra é considerada uma espécie invasora (HENDERSON, 2003; LOWE et al., 2004), com potencial de causar significativos danos ambientais e econômicos a biodiversidade de 
ecossistemas naturais (HEAR, 2005).

Diversas características biológicas da acácia-negra a capacitam como invasora de ambientes naturais, dentre os quais se destacam: floração aos 2 anos e grande produção de sementes a partir de 5 anos de idade (SHERRY, 1971; STEIN e TONIETTO, 1997), sementes apresentam dormência, permanecendo viáveis por longos períodos (MARTINS-CORDER et al., 1999; Pieterse e Boucher, 1997), queima e distúrbios no solo induzem germinação e o estabelecimento de plantas (PIETERSE e BOUCHER, 1997) e capacidade de adaptação a uma grande variedade de ambientes (KANNEGIESSER, 1990). No entanto, a dispersão das sementes da acácia-negra ocorre sobretudo por gravidade, estando limitada a pequenas distâncias (HEAR, 2005). É uma espécie exigente em luz direta para germinação de sementes e crescimento das plântulas, não invadindo áreas com solo coberto pela vegetação nativa (campos e florestas secundárias), desde de que não ocorra uma perturbação do ambiente que exponha o solo à plena luz (MOCHIUTTI et al., 2007).

O objetivo deste trabalho foi avaliar a regeneração natural de espécies florestais em um povoamento de acácia-negra estabelecido há 16 anos em uma área ripária e verificar a capacidade dessa espécie em manter uma população de plantas na área.

\section{MATERIAL E MÉTODOS}

\section{Área de estudo}

O estudo foi realizado na região da Encosta do Sudeste, município de Cristal, RS, situado entre as coordenadas geográficas de $31^{\circ} 07^{\prime}$ de latitude Sul e $52^{\circ} 03^{\prime}$ de longitude Oeste, a uma altitude em torno de $100 \mathrm{~m}$ acima do nível do mar. O clima da região, segundo a classificação de Köppen, é do tipo Cfa, com precipitações anuais em torno de $1.300 \mathrm{~mm}$, apresentando no ano déficit hídrico em 7 a $23 \mathrm{~mm}$, temperatura média de $18{ }^{\circ} \mathrm{C}$, mínima absoluta de $-5,0^{\circ} \mathrm{C}$ e 24 dias com geadas durante o ano (Rio Grande do Sul, 1994). A cobertura vegetal é formada pela transição entre a Floresta Estacional Semidecidual, Estepe Gramíneo-Lenhosa e Formações Pioneiras (Veloso et al., 1991; IBGE, 2004).

A área em estudo, com cerca de 1,0 ha, é uma pendente com aproximadamente $6 \%$ de inclinação, localizada dentro de uma área de preservação permanente e possuía cobertura original de mata de galeria, estando, em sua maior parte, circundada por florestas secundárias (Figura 1). Essa área foi utilizada por plantios de acácia-negra nas décadas 70 e 80 do século passado, sendo a última intervenção realizada em 1989, com a colheita e plantio de uma nova rotação de acácia-negra, no espaçamento de 1,5 x 3,0 m (2.222 plantas/ha). Esse plantio foi abandonado sem ser cortado, portanto o povoamento original de acácia-negra estava com 16 anos de idade quando foi realizado este estudo. $O$ solo da área caracteriza-se pela presença do horizonte B textural (Argissolo) a uma profundidade de $60 \mathrm{~cm}$ nos três quartos superiores da pendente e apresenta um aumento da umidade do solo na direção ao curso d'água.

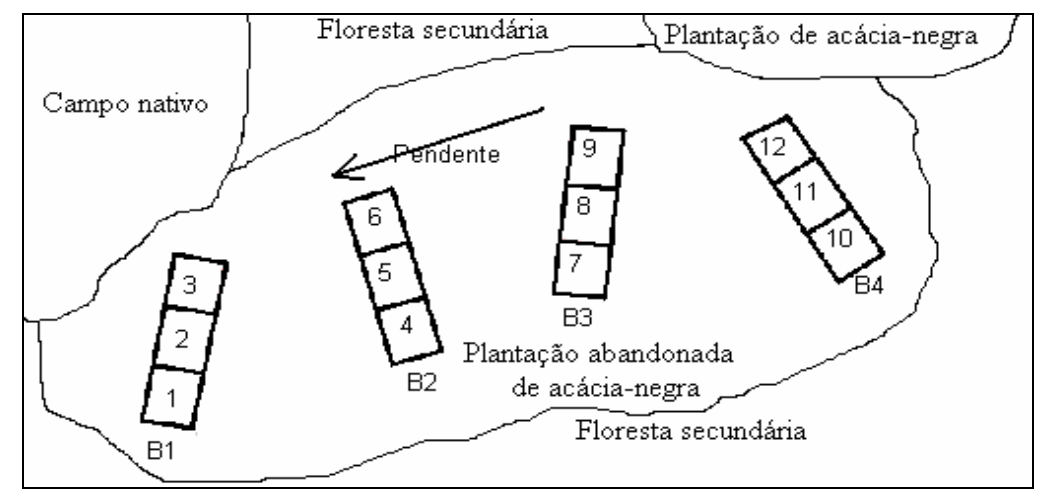

FIGURA 1: Localização dos blocos (B1, B2, B3 e B4) e parcelas (1 a 12) na plantação abandonada de acácia-negra, em Cristal, RS.

FIGURE 1: Location of the blocks (B1, B2, B3 and B4) and plots (1 to 12) in the abandoned black-wattle plantation, in Cristal, RS, Brazil. 


\section{Levantamento florístico e fitossociológico}

$\mathrm{O}$ estrato arbóreo (árvores com circunferência a $1,3 \mathrm{~m}$ de altura $-\mathrm{CAP} \geq 15 \mathrm{~cm}$ ) foi avaliado em 12 parcelas de $10 \times 10 \mathrm{~m}\left(100 \mathrm{~m}^{2}\right)$, totalizando $1.200 \mathrm{~m}^{2}$ ou $12 \%$ da área total. Essas parcelas foram demarcadas em quatro blocos de $10 \times 30 \mathrm{~m}$, contendo três parcelas cada um, dispostos transversalmente a maior pendente e alocados de maneira a abranger o gradiente de drenagem do solo existente na área (Figura 1). O bloco 1 foi localizado no quarto inferior da pendente (maior umidade) e o bloco 4 no quarto superior (menor umidade) e os blocos 2 e 3 intermediários a estes. A distância entre o bloco 1 (parte inferior) e o bloco 4 (parte superior) é de aproximadamente $150 \mathrm{~m}$. No levantamento do estrato arbóreo, foram medidas a altura e CAP de todos os indivíduos com CAP $\geq 15 \mathrm{~cm}$.

$\mathrm{O}$ estrato de regeneração natural (indivíduos de $0,3 \mathrm{~m}$ de altura a $\mathrm{CAP}<15 \mathrm{~cm}$ ) foi amostrada em duas subparcelas de $3 \times 3 \mathrm{~m}$, marcadas em vértices opostos de cada parcela do levantamento do estrato arbóreo. $\mathrm{O}$ estrato de regeneração natural foi dividido em três classes de altura: $\mathrm{I}=0,3$ a $1,0 \mathrm{~m}$ de altura, $\mathrm{II}=$ $1,0 \mathrm{~m}$ a $3,0 \mathrm{~m}$ de altura e III $=3,0 \mathrm{~m}$ de altura a CAP $<15 \mathrm{~cm}$. A classe de altura I foi avaliada em uma das subparcelas $\left(9 \mathrm{~m}^{2}\right)$ e as classes II e III nas duas subparcelas $\left(18 \mathrm{~m}^{2}\right)$. Foi anotada a altura de todos os indivíduos de espécies arbóreas.

A identificação das espécies foi realizada por meio de material botânico coletado, contou com o auxilio de especialistas do Departamento de Botânica da UFRGS e do Jardim Botânico de Curitiba, e de comparações de exsicatas dos herbários dessas duas instituições. A confirmação da grafia das espécies e nome dos respectivos autores foi feita pelo site do Missouri Botanical Garden (www.mobot.org).

\section{Análise de dados}

$\mathrm{Na}$ análise fitossociológica dos estratos arbóreo e de regeneração natural (total e nas três classes de altura) foram calculados, no software Microsoft Excel 2002, os seguintes parâmetros: porcentagem de cobertura por família e densidade, freqüência, dominância e valor de importância por espécie, conforme Mueller-Dombois e Ellenberg (1974). Também foi estimada a diversidade específica dos estratos arbóreo e de regeneração natural, pelo índice de Shannon ( $\left.\mathrm{H}^{\prime}\right)$, utilizando-se a base logarítmica natural, sendo os resultados expressos em nats/indivíduos (MAGURRAN, 1988).

Para a análise da estrutura vertical foi elaborado um histograma com a distribuição dos indivíduos encontrados em oito classes de altura, com intervalo de $3 \mathrm{~m}$, apresentados graficamente pelo logaritmo natural do número de indivíduos/ha. Para essa análise, foram definidas quatro posições sociológicas: estrato superior (18 a $24 \mathrm{~m}$ de altura), intermediário (12 a $15 \mathrm{~m}$ de altura), inferior (6 a $12 \mathrm{~m}$ de altura) e de regeneração natural ( 0,3 a $6 \mathrm{~m}$ de altura).

As espécies encontradas foram agrupadas em três síndromes de dispersão (anemocórica, autocórica e zoocórica), com base nas características dos frutos e sementes; e três grupos ecológico (pioneira, clímax exigente de luz e clímax tolerantes a sombra), definidos segundo a classificação de Swaine e Whitmore (1988), modificada por Oliveira Filho (1994). A classificação das espécies por categoria de dispersão e grupo ecológico baseou-se em dados da literatura, notadamente Lorenzi (1992 e 1998), Carvalho (1994, 2003 e 2006), Backes e Irgang (2002), Reitz et al. (1988) e em observações diretas e experiência de campo dos autores.

A classificação de espécies em grupos ecológicos é dificultada pelos diferentes critérios utilizados por diversos autores, o que leva uma mesma espécie ser classificada em grupos distintos, bem como, ao fato de que uma espécie, dependendo de suas características genéticas, pode apresentar diferentes comportamentos diante de condições ambientais distintas, uma vez que essas respostas não se dão para um único fator do meio isoladamente.

\section{RESULTADOS E DISCUSSÃO}

\section{Composição e diversidade florística}

Na Tabela 1, está apresentada a relação das espécies encontradas, em ordem alfabética por família e gênero, com respectivos grupos ecológicos, tipos de dispersão e número de indivíduos amostrados nos estratos arbóreo e de regeneração natural. Uma síntese das informações dos parâmetros fitossociológicos é apresentada na Tabela 2. Houve suficiência amostral, conforme demonstrado na Figura 2, na qual se verifica 
a estabilização da curva do número de espécies partindo da parcela 8 , tanto do estrato arbóreo como do estrato de regeneração natural.

No estrato arbóreo (árvores com CAP $\geq 15 \mathrm{~cm}$ ) foram encontradas 26 espécies nativas pertencentes a 14 famílias botânicas (Tabela 2). O valor do índice de diversidade de Shannon para espécies foi de $\mathrm{H}^{\prime}=$ 2,60, considerando as parcelas como um todo, e de 1,70 para a média das parcelas, com variação entre 1,01 a 2,38. As famílias com o maior número de espécies no estrato arbóreo foram Flacourtiaceae, Myrsinaceae e Myrtaceae que apresentaram três espécies cada.

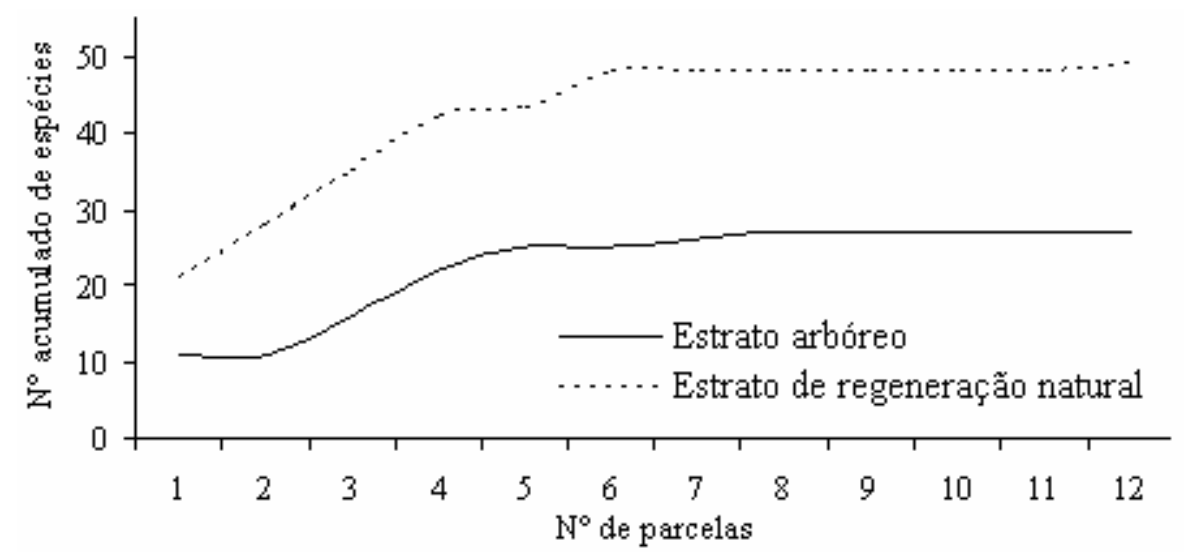

FIGURA 2: Curva cumulativa das espécies amostradas na regeneração natural de um povoamento de acácianegra, em Cristal, RS.

FIGURE 2: Species area curve sampled in the natural regeneration of a black-wattle plantation, in Cristal, RS, Brazil.

No estrato de regeneração natural (indivíduos de $0,30 \mathrm{~m}$ de altura a $\mathrm{CAP}<15 \mathrm{~cm}$ ), verificou-se uma redução do número de indivíduos, espécies e famílias amostradas e do índice de diversidade, à medida que se aumentou a classe de altura das plantas (Tabela 2). Considerando o total das três classes de altura do estrato de regeneração natural, foram encontradas 49 espécies distribuídas em 23 famílias botânicas (Tabela 2). O índice de diversidade de espécies, para as parcelas como um todo, foi de $\mathrm{H}^{\prime}=3,03$ e nas parcelas variou de 1,75 a 2,81, com média de 2,33. A família Myrtaceae apresentou maior riqueza, com dez espécies no estrato de regeneração natural, seguida pelas famílias Euphorbiaceae, Flacourtiaceae, Lauraceae, Meliaceae, Myrsinaceae e Sapindaceae, com três espécies cada.

No inventário florestal contínuo do Rio Grande do Sul da região da Floresta Estacional Semidecidual, mesma região fitogeográfica deste trabalho, verificou-se que os estágios iniciais da sucessão secundária apresentaram 41 espécies pertencentes a 25 famílias botânicas e índice de diversidade de H' = 1,74, tendo como critério de inclusão plantas com CAP $\geq 3,0 \mathrm{~cm}$ (SEMA, 2001). Na regeneração natural (CAP entre 3 e $30 \mathrm{~cm}$ ) de estágios sucessionais médio e avançado do mesmo inventário encontraram-se 58 espécies de 26 famílias botânicas, com índice de diversidade de $H^{\prime}=1,80$. Entre as vinte espécies mais abundantes encontradas no inventário, dez espécies aparecem entre as de maior freqüência na regeneração natural do presente trabalho.

A diversidade da regeneração natural citada para diversas plantações florestais é de difícil comparação, por causa das diferenças metodológicas, como a área amostrada, limites para a inclusão de indivíduos, tamanho e idade do povoamento, fitogeografia da região, base logarítmica utilizada, etc. No entanto, a maioria dos valores de diversidade encontrados na literatura indica que essas plantações não impedem o desenvolvimento de espécies nativas em seu sub-bosque.

Para povoamentos de Eucalyptus spp., Sartori et al. (2002) citam valores de H' entre 0,76 a 3,75. No Rio Grande do Sul, Andrae et al. (2005) encontraram variações de H' entre 1,3 a 3,5 para plantações de Pinus spp. em pequenas propriedades. A regeneração natural em sub-bosque de Mimosa scabrella apresentou H'=2,85 (NAPPO et al., 2000) e em sub-bosque de três leguminosas arbóreas H' variou entre 1,90 e 2,59 (CHADA et al., 2004). 
TABELA 1: Lista das espécies nativas e número de indivíduos amostrados nos estratos arbóreo e de regeneração natural de um povoamento de acácia-negra, em Cristal, RS.

TABLE 1: List of native species and number of individuals sampled in an tree and natural regeneration strata sampled in a black-wattle plantation, in Cristal, RS, Brazil.

\begin{tabular}{|c|c|c|c|c|c|c|c|c|c|c|}
\hline \multirow{3}{*}{ Família/Espécie } & \multirow{3}{*}{ GE } & \multirow{3}{*}{ Disp. } & \multicolumn{8}{|c|}{ Estratos } \\
\hline & & & \multicolumn{4}{|c|}{ Arbóreo } & \multicolumn{4}{|c|}{ Regeneração natural } \\
\hline & & & B1 & B2 & B3 & B4 & $\mathrm{B} 1$ & $\mathrm{~B} 2$ & B3 & B4 \\
\hline \multicolumn{11}{|l|}{ Anacardiaceae } \\
\hline Lithraea brasiliensis March. & CL & Zoo & & 1 & & & & 1 & & \\
\hline Schinus terebinthifolius Raddi & $\mathrm{P}$ & Zoo & 1 & & & & 2 & & & \\
\hline \multicolumn{11}{|l|}{ Arecaceae } \\
\hline Syagrus romanzoffiana (Cham.) Glassm. & $\mathrm{CL}$ & Zoo & & & & & & 1 & 2 & \\
\hline \multicolumn{11}{|l|}{ Asteraceae } \\
\hline Dasyphyllum spinescens (Less.) Cabrera & - & Ane & & & & & 1 & 1 & & \\
\hline Gochnatia polymorpha (Less.) Cabrera & $\mathrm{P}$ & Ane & 5 & 2 & 3 & 5 & & & 3 & 2 \\
\hline \multicolumn{11}{|l|}{ Bignoniaceae } \\
\hline Tabebuia heptaphylla (Vell.) Tol. & $\mathrm{CL}$ & Ane & & & & & & 2 & 1 & 2 \\
\hline \multicolumn{11}{|l|}{ Boraginaceae } \\
\hline Cordia ecalyculata Vell. & $\mathrm{CL}$ & Zoo & & & 1 & & 4 & & 4 & 1 \\
\hline Patagonula americana L. & $\mathrm{CL}$ & Ane & & 1 & & & & 1 & 3 & 6 \\
\hline \multicolumn{11}{|l|}{ Celtidaceae } \\
\hline Trema micrantha (L.) Blume & $\mathrm{P}$ & Zoo & & & & & 2 & & & \\
\hline \multicolumn{11}{|l|}{ Euphorbiaceae } \\
\hline Alchornea triplinervia (Spreng.) Müll. Arg. & $\mathrm{CL}$ & Zoo & 1 & & & & 1 & & & \\
\hline Gymnanthes concolor (Spreng.) Müll. Arg. & $\mathrm{CS}$ & Aut & & & & & 1 & & & \\
\hline Sebastiania brasiliensis Spreng. & $\mathrm{CS}$ & Aut & & & & & 1 & & & \\
\hline \multicolumn{11}{|l|}{ Fabaceae } \\
\hline Inga sp. & - & Zoo & & & & & 3 & & & \\
\hline \multicolumn{11}{|l|}{ Flacourtiaceae } \\
\hline Banara sp. & - & Zoo & 3 & & & & 3 & & & 1 \\
\hline Casearia decandra Jacq. & $\mathrm{CL}$ & Zoo & 4 & 4 & 3 & 1 & 10 & 17 & 11 & 5 \\
\hline Casearia sylvestris Sw. & $\mathrm{P}$ & Zoo & 11 & 18 & 10 & 8 & 8 & 7 & 12 & 9 \\
\hline \multicolumn{11}{|l|}{ Lauraceae } \\
\hline Nectandra lanceolata Nees & $\mathrm{CS}$ & Zoo & & & & & 3 & & 3 & 1 \\
\hline Ocotea puberula (Rich.) Nees & $\mathrm{CL}$ & Zoo & 7 & & & & 4 & 2 & 4 & 1 \\
\hline Ocotea pulchella (Nees) Mez & $\mathrm{CL}$ & Zoo & 1 & & & & 8 & 6 & 2 & 2 \\
\hline \multicolumn{11}{|l|}{ Melastomataceae } \\
\hline Miconia sp. & - & Zoo & & & & & 2 & 1 & & \\
\hline \multicolumn{11}{|l|}{ Meliaceae } \\
\hline Cabralea canjerana (Vell.) Mart. & $\mathrm{CS}$ & Zoo & 4 & & & & 4 & & & \\
\hline Cedrela fissilis Vell. & $\mathrm{CL}$ & Ane & & & & & & 2 & 1 & \\
\hline Trichilia claussenii C. DC. & $\mathrm{CS}$ & Zoo & & 1 & & & 1 & 3 & 1 & \\
\hline \multicolumn{11}{|l|}{ Moraceae } \\
\hline $\begin{array}{l}\text { Sorocea bonplandii (Baill.) W.C. Burger, Lanj. \& } \\
\text { Wess. Boer }\end{array}$ & $\mathrm{CS}$ & Zoo & & & & & 2 & & & \\
\hline \multicolumn{11}{|l|}{ Myrsinaceae } \\
\hline $\begin{array}{l}\text { Myrsine coriacea (Sw.) R. Br. ex Roem. \& } \\
\text { Schult. }\end{array}$ & $\mathrm{P}$ & Zoo & 1 & & 1 & & 2 & 5 & 6 & 11 \\
\hline Myrsine lorentziana (Mez) Arechav. & $\mathrm{CL}$ & Zoo & 9 & 2 & 8 & 1 & 13 & 34 & 32 & 17 \\
\hline Myrsine umbellata Mart. & $\mathrm{CL}$ & Zoo & & & 1 & 6 & & & 7 & 23 \\
\hline
\end{tabular}


TABELA 1: Continuação ...

TABLE 1: Continued ...

\begin{tabular}{|c|c|c|c|c|c|c|c|c|c|c|}
\hline \multirow{3}{*}{ Família/Espécie } & \multirow{3}{*}{ GE } & \multirow{3}{*}{ Disp. } & \multicolumn{8}{|c|}{ Estratos } \\
\hline & & & \multicolumn{4}{|c|}{ Arbóreo } & \multicolumn{4}{|c|}{ Regeneração natural } \\
\hline & & & B1 & B2 & B3 & B4 & B1 & B2 & B3 & B4 \\
\hline \multicolumn{11}{|l|}{ Myrtaceae } \\
\hline Blepharocalyx salicifolius (Kunth) O. Berg & $\mathrm{CL}$ & Zoo & & & & & & 1 & 6 & 1 \\
\hline Eugenia uniflora L. & $\mathrm{CL}$ & Zoo & & 1 & & & & 5 & 1 & 3 \\
\hline Eugenia sp. & & Zoo & & & & & 4 & & & \\
\hline Myrcia glabra (O. Berg) D. Legrand & $\mathrm{CS}$ & Zoo & 5 & & & 2 & 50 & 12 & 6 & 2 \\
\hline Myrcia sp. & 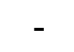 & Zoo & & 1 & & & 4 & & 1 & \\
\hline Myrcianthes gigantea (D. Legrand) D. Legrand & $\mathrm{CL}$ & Zoo & & & & & 6 & 3 & & \\
\hline Myrcianthes pungens (O. Berg) D. Legrand & $\mathrm{CS}$ & Zoo & & & & & 1 & & & \\
\hline Myrciaria tenella (DC.) O. Berg & $\mathrm{CL}$ & Zoo & & & & & & & 2 & \\
\hline Psidium cattleyanum Sabine & $\mathrm{P}$ & Zoo & & & & & & 2 & & \\
\hline Myrtaceae 1 & - & Zoo & & & & & 3 & & & \\
\hline \multicolumn{11}{|l|}{ Rosaceae } \\
\hline Prunus sellowii Koehne & CL & Zoo & 1 & & & & 2 & & 1 & 1 \\
\hline \multicolumn{11}{|l|}{ Rubiaceae } \\
\hline Faramea marginata Cham. & $\mathrm{CS}$ & Zoo & & & & & 96 & 3 & 7 & \\
\hline Guettarda uruguensis Cham. \& Schltdl. & $\mathrm{CS}$ & Zoo & & & & & 1 & & & \\
\hline \multicolumn{11}{|l|}{ Rutaceae } \\
\hline Zanthoxylum hyemale A. St.-Hil. & $P$ & Zoo & & 5 & & & & & & 1 \\
\hline Sapindaceae & $\mathrm{CL}$ & Zoo & 2 & 12 & 6 & & 4 & 5 & 2 & 6 \\
\hline $\begin{array}{l}\text { Allophylus edulis (A. St.-Hil., Cambess. \& A. } \\
\text { Juss.) Radlk. }\end{array}$ & $\mathrm{P}$ & Zoo & 2 & & & & 2 & 3 & 9 & 5 \\
\hline Cupania vernalis Cambess. & $\mathrm{CL}$ & Zoo & & & & & & 4 & & 3 \\
\hline Matayba elaeagnoides Radlk. & CL & Zoo & 4 & & & & 5 & & 1 & \\
\hline \multicolumn{11}{|l|}{ Symplocaceae } \\
\hline Symplocos uniflora (Pohl) Benth. & $\mathrm{CS}$ & Zoo & 1 & 2 & & & 3 & & & 3 \\
\hline \multicolumn{11}{|l|}{ Thymelaeaceae } \\
\hline Daphnopsis racemosa Griseb. & $\mathrm{CS}$ & Zoo & & & & & 22 & 3 & 2 & 2 \\
\hline Tiliaceae & & & & & & & & & & \\
\hline Luehea divaricata Mart. & CL & Ane & & 1 & & & & 7 & 1 & \\
\hline Verbenaceae & & & & & & & & & & \\
\hline Vitex megapotamica (Spreng.) Moldenke & $\mathrm{CL}$ & Zoo & & & & & 1 & & & \\
\hline
\end{tabular}

Em que: $\mathrm{GE}=$ grupo ecológico; $\mathrm{P}=$ pioneira; $\mathrm{CL}=$ clímax exigente de luz; $\mathrm{CS}=$ clímax tolerantes à sombra; Disp. $=$ tipo de dispersão; Ane = anemocórica; Aut = autocórica; Zoo = Zoocórica; B1, B2, B3, B4 = respectivamente os blocos $1,2,3$ e 4. 
TABELA 2: Parâmetros fitossociológicos dos estratos arbóreo e de regeneração natural obtidos em um povoamento de acácia-negra, em Cristal, RS.

TABLE 2: Phytosociological parameters of the tree and natural regeneration strata obtained in a black-wattle plantation, in Cristal, RS, Brazil.

\begin{tabular}{lcr|rrrr}
\hline \multirow{2}{*}{ Parâmetros } & \multirow{2}{*}{$\begin{array}{c}\text { Estrato } \\
\text { arbóreo }\end{array}$} & \multicolumn{4}{c}{ Estrato de regeneração natural $^{2}$} \\
\cline { 3 - 6 } & & I & II & III & Total \\
\hline Densidade das espécies nativas (ind/ha) & 1.408 & 38.241 & 8.565 & 2.361 & 49.167 \\
Densidade da acácia-negra (ind/ha) & 225 & 0 & 0 & 0 & 0 \\
Área basal das espécies nativas (m/2/ha) & 9,66 & - & - & - & - \\
Área basal da acácia-negra $\left(\mathrm{m}^{2} / \mathrm{ha}^{3}\right.$ & 9,39 & - & - & - & - \\
Indivíduos amostrados de espécies nativas & 169 & 413 & 185 & 51 & 649 \\
Número de famílias na sucessão natural & 14 & 22 & 17 & 12 & 23 \\
Número de espécies na sucessão natural & 26 & 45 & 34 & 18 & 49 \\
Índice de diversidade de Shannon (H') & 2,60 & 2,95 & 2,93 & 2,51 & 3,03 \\
\hline
\end{tabular}

Em que: 1 = Árvores com CAP $\geq 15,0 \mathrm{~cm} ; 2$ = Classes de altura: I ( 0,3 a 1,0 m), II $(1,0$ a 3,0 m) e III $(3,0 \mathrm{~m}$ de altura e até $\mathrm{CAP}<15 \mathrm{~cm}) ; 3=$ Plantas vivas + mortas em pé.

Apesar da dificuldade de comparações de índices de diversidade, os valores encontrados para parcelas individuais neste trabalho são similares aos reportados para a regeneração natural de estágios sucessionais iniciais, médio e avançado da Floresta Estacional Semidecidual do Rio Grande do Sul (SEMA, 2001) e estão acima da média aos citados para sub-bosques de diversos povoamentos florestais, considerando as parcelas como um todo.

\section{Estrutura dos estratos arbóreo e de regeneração natural}

No estrato arbóreo (árvores com CAP $\geq 15 \mathrm{~cm}$ ) foram mensurados 169 indivíduos de espécies nativas, que representam uma densidade estimada de $1.408 \mathrm{ind} / \mathrm{ha}$ e uma área basal de $9,66 \mathrm{~m}^{2} / \mathrm{ha}$ (Tabela 2). Foram encontradas somente cem árvores vivas/ha de acácia-negra, que representam apenas $4,5 \%$ da densidade de plantio do povoamento. As plantas mortas em pé de acácia-negra totalizaram $125 \mathrm{ind} / \mathrm{ha}$, sendo verificada uma grande quantidade de árvores mortas caídas (não-avaliadas).

A Acacia mearnsii (Fabaceae) apresentou a maior porcentagem de cobertura $(33,4 \%)$ do estrato arbóreo, seguida pelas famílias Flacourtiaceae $(24,5 \%)$, Myrsinaceae $(11,7 \%)$ e Rutaceae $(10,3 \%)$, as quais participaram com $79,9 \%$ da cobertura total. As dez espécies que tiveram os maiores valores de importância no estrato arbóreo foram: Casearia sylvestris Sw. (53,6), Acacia mearnsii - plantas vivas $(43,1)$, Acacia mearnsii - plantas mortas (40,3), Myrsine lorentziana (Mez) Arechav. (24,7), Zanthoxylum petiolare A. St.-Hil. \& Tul. (24,6), Gochnatia polimorpha (Less.) Cabrera $(17,4)$, Casearia decandra Jacq. $(13,9)$, Myrsine umbellata Mart. (11,3), Ocotea puberula (Rich.) Nees $(9,0)$ e Myrcia glaba (O. Berg) D. Legrand $(7,9)$. A contribuição dessas espécies resultou em $81,9 \%$ da soma total do valor de importância (Tabela 3). A Casearia sylvestris apresentou a maior densidade e freqüência, enquanto que a Acacia mearnsii a maior dominância, pelo maior diâmetro das árvores.

As famílias com maior porcentagem de cobertura no estrato de regeneração natural (indivíduos de $0,3 \mathrm{~m}$ de altura a CAP $<15 \mathrm{~cm}$ ) foram Myrsinaceae (23,3\%), Myrtaceae (17,1\%), Rubiaceae (16,3\%) e Flacourtiaceae $(13,4 \%)$; a participação dessas famílias representou $70,1 \%$ da cobertura total. Na classe de altura I $(0,3 \mathrm{~m}$ a $1,0 \mathrm{~m})$ do estrato de regeneração natural foi estimada uma densidade total de $38.241 \mathrm{ind} / \mathrm{ha}$ (Tabela 2), tendo destaque as seguintes espécies: Faramea marginata Cham., Myrsine lorentziana, Myrsia glabra, Casearia decandra, Daphnopsis racemosa Griseb., Myrsine coriacea (Sw.) R. Br. ex Roem. \& Schult., Allophylus edulis (A. St.-Hil., Cambess. \& A. Juss.) Radlk., Casearia sylvestris e Ocotea pulchella (Nees) Mez (Tabela 4). Unidas, estas espécies representam 71,7\% da densidade total. 
TABELA 3: Estrutura do estrato arbóreo em um povoamento de acácia-negra, em Cristal, RS.

TABLE 3: Structure of the tree stratum in a black-wattle plantation, in Cristal, RS, Brazil.

\begin{tabular}{|c|c|c|c|c|c|c|}
\hline Espécies & $\begin{array}{l}\text { DAP } \\
(\mathrm{cm})\end{array}$ & $\begin{array}{c}\mathrm{h} \\
(\mathrm{m})\end{array}$ & $\begin{array}{l}\text { FA } \\
(\%)\end{array}$ & $\begin{array}{c}\text { DA } \\
\text { (ind/ha) }\end{array}$ & $\begin{array}{c}\text { DoA } \\
\left(\mathrm{m}^{2} / \mathrm{ha}\right)\end{array}$ & VI \\
\hline Casearia sylvestris & 9,2 & 6,6 & 100 & 392 & 2,9 & 53,6 \\
\hline Acacia mearnsii - plantas vivas & 25,3 & 19,5 & 58 & 100 & 5,4 & 43,1 \\
\hline Acacia mearnsii - plantas mortas & 17,9 & - & 67 & 125 & 4,0 & 40,3 \\
\hline Myrsine lorentziana & 7,8 & 6,8 & 75 & 167 & 0,9 & 24,7 \\
\hline Zanthoxylum petiolare & 8,4 & 7,4 & 58 & 167 & 1,2 & 24,6 \\
\hline Gochnatia polymorpha & 10,4 & 5,9 & 42 & 125 & 1,3 & 17,4 \\
\hline Casearia decandra & 6,1 & 6,7 & 50 & 100 & 0,3 & 13,9 \\
\hline Myrsine umbellata & 7,1 & 6,1 & 33 & 58 & 0,3 & 11,3 \\
\hline Ocotea puberula & 10,8 & 9,3 & 25 & 58 & 0,5 & 9,0 \\
\hline Myrcia glabra & 5,9 & 6,5 & 33 & 58 & 0,2 & 7,9 \\
\hline Zanthoxylum hyemale & 8,3 & 7,6 & 25 & 42 & 0,2 & 6,9 \\
\hline Cabralea canjerana & 14,6 & 10,3 & 17 & 33 & 0,6 & 6,5 \\
\hline Matayba elaeagnoides & 7,4 & 8,2 & 25 & 33 & 0,1 & 5,6 \\
\hline Symplocos uniflora & 10,1 & 6,8 & 17 & 25 & 0,2 & 4,5 \\
\hline Myrsine coriacea & 5,6 & 6,5 & 17 & 17 & 0,0 & 3,8 \\
\hline Banara sp. & 5,0 & 6,8 & 17 & 25 & 0,1 & 3,5 \\
\hline Alchornea triplinervia & 21,2 & 10,0 & 8 & 8 & 0,3 & 3,0 \\
\hline Prunus sellowii & 21,0 & 14,0 & 8 & 8 & 0,3 & 2,9 \\
\hline Allophylus edulis & 5,4 & 5,5 & 8 & 17 & 0,0 & 2,3 \\
\hline Cordia ecalyculata & 4,8 & 4,5 & 8 & 8 & 0,0 & 1,9 \\
\hline Myrcia sp. & 7,0 & 7,0 & 8 & 8 & 0,0 & 1,8 \\
\hline Ocotea pulchella & 6,4 & 4,5 & 8 & 8 & 0,0 & 1,8 \\
\hline Trichilia claussenii & 7,2 & 9,0 & 8 & 8 & 0,0 & 1,7 \\
\hline Eugenia uniflora & 6,1 & 6,0 & 8 & 8 & 0,0 & 1,7 \\
\hline Lithraea brasiliensis & 6,0 & 5,0 & 8 & 8 & 0,0 & 1,7 \\
\hline Luehea divaricata & 4,8 & 4,0 & 8 & 8 & 0,0 & 1,7 \\
\hline Patagonula americana & 5,4 & 6,0 & 8 & 8 & 0,0 & 1,6 \\
\hline Schinus terebinthifolius & 5,9 & 8,0 & 8 & 8 & 0,0 & 1,5 \\
\hline
\end{tabular}

Em que: DAP $=$ diâmetro médio a $1,30 \mathrm{~m}$ de altura; $\mathrm{h}=$ altura média; $\mathrm{FA}=$ freqüência absoluta; $\mathrm{DA}=$ densidade absoluta; DoA = dominância absoluta; VI = valor de importância.

A densidade total estimada da classe de altura II $(1,0$ a 3,0 m) foi de $8.565 \mathrm{ind} / \mathrm{ha}$, merecendo destacar as seguintes espécies: Myrsine lorentziana, Myrcia glabra, Casearia sylvestris, Myrsine umbellata, Faramea marginata, Casearia decandra, Daphnopsis racemosa e Zanthoxylum petiolare, que contribuíram com $68,7 \%$ da densidade total. Na classe de altura III $(3,0 \mathrm{~m}$ de altura a CAP $<15,0 \mathrm{~cm})$, a densidade total foi estimada em $2.361 \mathrm{ind} /$ ha. Representando $70,6 \%$ dessa densidade estão as seguintes espécies: Casearia decandra, Myrsine umbellata, Myrsine lorentziana, Casearia sylvestris, Myrcia glaba e Zanthoxylum petiolare.

Considerando o estrato de regeneração natural como um todo (indivíduos de $0,3 \mathrm{~m}$ de altura a CAP $>15,0 \mathrm{~cm}$ ), as dez espécies de maior densidade foram: Faramea marginata, Myrsine lorentziana, Myrsia glabra, Casearia decandra, Daphnopsis racemosa, Casearia sylvestris, Myrsine coriacea, Myrsine umbellata, Allophylus edulis e Ocotea pulchella, que representam juntas $72,8 \%$ da densidade total (Tabela 4). 
TABELA 4: Densidade e freqüência absoluta do estrato de regeneração natural em um povoamento de acácia-negra, em Cristal, RS.

TABLE 4: Absolute density and frequency of the natural regeneration stratum in a black-wattle plantation, in Cristal, RS, Brazil.

\begin{tabular}{|c|c|c|c|c|c|}
\hline \multirow[t]{2}{*}{ Espécie } & \multicolumn{4}{|c|}{ Densidade (ind/ha) } & \multirow{2}{*}{$\begin{array}{c}\text { Freqüência } \\
(\%)\end{array}$} \\
\hline & Classe I $^{1}$ & Classe II & Classe III & Total & \\
\hline Faramea marginata & 8.426 & 602 & 93 & 9.120 & 66,7 \\
\hline Myrsine lorentziana & 5.000 & 1.667 & 278 & 6.944 & 100,0 \\
\hline Myrcia glabra & 4.352 & 926 & 139 & 5.417 & 91,7 \\
\hline Casearia decandra & 2.222 & 417 & 463 & 3.102 & 75,0 \\
\hline Daphnopsis racemosa & 1.852 & 417 & 0 & 2.269 & 66,7 \\
\hline Casearia sylvestris & 1.204 & 833 & 231 & 2.269 & 91,7 \\
\hline Myrsine coriacea & 1.852 & 139 & 46 & 2.037 & 83,3 \\
\hline Myrsine umbellata & 556 & 694 & 417 & 1.667 & 41,7 \\
\hline Allophylus edulis & 1.389 & 139 & 46 & 1.574 & 66,7 \\
\hline Ocotea pulchella & 1.111 & 185 & 93 & 1.389 & 58,3 \\
\hline Zanthoxylum petiolare & 648 & 324 & 139 & 1.111 & 83,3 \\
\hline Ocotea puberula & 833 & 93 & 0 & 926 & 66,7 \\
\hline Cordia ecalyculata & 741 & 46 & 0 & 787 & 33,3 \\
\hline Myrcianthes gigantea & 556 & 139 & 0 & 694 & 41,7 \\
\hline Eugenia uniflora & 556 & 139 & 0 & 694 & 50,0 \\
\hline Cupania vernalis & 556 & 46 & 0 & 602 & 33,3 \\
\hline Patagonula americana & 278 & 231 & 93 & 602 & 33,3 \\
\hline Blepharocalyx salicifolius & 463 & 139 & 0 & 602 & 33,3 \\
\hline Nectandra lanceolata & 463 & 93 & 0 & 556 & 50,0 \\
\hline Luehea divaricata & 278 & 185 & 46 & 509 & 33,3 \\
\hline Tabebuia heptaphylla & 463 & 0 & 0 & 463 & 33,3 \\
\hline Gochnatia polymorpha & 370 & 0 & 46 & 417 & 25,0 \\
\hline Matayba elaeagnoides & 278 & 139 & 0 & 417 & 33,3 \\
\hline Symplocos uniflora & 278 & 139 & 0 & 417 & 25,0 \\
\hline Banara sp. & 278 & 46 & 0 & 324 & 16,7 \\
\hline Prunus sellowii & 278 & 46 & 0 & 324 & 25,0 \\
\hline Eugenia sp. & 278 & 46 & 0 & 324 & 8,3 \\
\hline Myrtaceae 1 & 278 & 0 & 0 & 278 & 16,7 \\
\hline Cabralea canjerana & 185 & 46 & 46 & 278 & 16,7 \\
\hline Syagrus romanzoffiana & 278 & 0 & 0 & 278 & 25,0 \\
\hline Myrcia sp. & 93 & 139 & 46 & 278 & 25,0 \\
\hline Trichilia claussenii & 93 & 185 & 0 & 278 & 25,0 \\
\hline Inga sp. & 185 & 46 & 0 & 231 & 25,0 \\
\hline Psidium cattleyanum & 185 & 0 & 0 & 185 & 8,3 \\
\hline Schinus terebinthifolius & 185 & 0 & 0 & 185 & 8,3 \\
\hline Cedrela fissilis & 93 & 46 & 46 & 185 & 16,7 \\
\hline Sorocea bonplandii & 185 & 0 & 0 & 185 & 8,3 \\
\hline Trema micrantha & 185 & 0 & 0 & 185 & 16,7 \\
\hline Dasyphyllum spinescens & 185 & 0 & 0 & 185 & 16,7 \\
\hline Myrciaria tenella & 93 & 0 & 46 & 139 & 16,7 \\
\hline Miconia sp. & 0 & 93 & 46 & 139 & 25,0 \\
\hline Zanthoxylum hyemale & 93 & 0 & 0 & 93 & 8,3 \\
\hline Myrcianthes pungens & 93 & 0 & 0 & 93 & 8,3 \\
\hline
\end{tabular}


TABELA 1: Continuação ...

TABLE 1: Continued ...

\begin{tabular}{l|c|c|c|c|c}
\hline \multirow{2}{*}{ Espécie } & \multicolumn{4}{|c|}{ Densidade (ind/ha) } & \multirow{2}{*}{$\begin{array}{c}\text { Freqüência } \\
\text { (\%) }\end{array}$} \\
\cline { 2 - 5 } & Classe I $^{1}$ & Classe II & Classe III & Total & 8,3 \\
Gymnanthes concolor & 93 & 0 & 0 & 93 & 8,3 \\
Guettarda uruguensis & 93 & 0 & 0 & 93 & 8,3 \\
Vitex megapotamica & 93 & 0 & 0 & 93 & 8,3 \\
Lithraea brasiliensis & 0 & 46 & 0 & 46 & 8,3 \\
Sebastiania brasiliensis & 0 & 46 & 0 & 46 & 8,3 \\
Alchornea triplinervia & 0 & 46 & 0 & 46 & \\
\hline
\end{tabular}

Em que: $1=$ Classe $(\mathrm{I}=0,3$ a $1,0 \mathrm{~m}$ de altura; Classe $\mathrm{II}=1,0$ a $3,0 \mathrm{~m}$ de altura; Classe $\mathrm{III}=3,0 \mathrm{~m}$ de altura a $<15 \mathrm{~cm}$ de CAP).

A densidade total de $49.167 \mathrm{ind} / \mathrm{ha}$ no estrato de regeneração natural observada neste trabalho é superior à relatada em outras plantações florestais. Chada et al. (2004) encontraram uma densidade de 2.915 ind/ha na regeneração natural de um reflorestamento com leguminosas arbóreas, tendo como critério de inclusão indivíduos com altura a partindo de $0,4 \mathrm{~m}$. No sub-bosque de Minosa scabrella em área minerada foram encontrados $30.410 \mathrm{ind} / \mathrm{ha}$ com altura $\geq 0,3 \mathrm{~m}$ (NAPPO et al., 2004). A densidade da regeneração natural de uma clareira da Floresta Estacional Semidecídua foi de $36.136 \mathrm{ind} / \mathrm{ha}$, também com altura $\geq 0,3 \mathrm{~m}$ (RONDON NETO et al., 2000).

A maior densidade da regeneração natural encontrada neste trabalho pode ser atribuída à melhoria das condições ambientais do sub-bosque proporcionadas pela deposição de grande quantidade de serapilheira rica em nutrientes, especialmente o nitrogênio (SCHUMACHER et al., 2003); abertura de clareiras pela mortalidade e queda da maioria das árvores de acácia-negra que já completaram seu ciclo de vida; bem como, boa disponibilidade de propágulos. Dessa forma, a acácia-negra pode ser considerada como uma espécie facilitadora da regeneração natural, podendo ser utilizada na recuperação da cobertura florestal de áreas em que a vegetação nativa tenha dificuldade de regeneração, em conseqüência de condições desfavoráveis de solo.

Quanto à análise da estrutura vertical, foram definidas quatro posições sociológicas (Figura 3). O estrato superior, com altura de 18 a $24 \mathrm{~m}$, é formado exclusivamente pela acácia-negra (100 ind/ha), árvores remanescentes do plantio original. O estrato intermediário, com 12 a $15 \mathrm{~m}$ de altura, agrupou poucos indivíduos de Prumus sellowii Koehne, Casearia sylvestris e Ocotea puberula, bem dispersos na área ( $25 \mathrm{ind} / \mathrm{ha}$ ), não sendo encontradas árvores com altura entre 15 e $18 \mathrm{~m}$. O estrato inferior, com altura de 6 a $12 \mathrm{~m}$, concentrou a maior parte das plantas $(1.631 \mathrm{ind} / \mathrm{ha})$ do estrato arbóreo (CAP $\geq 15,0 \mathrm{~cm})$, sendo formado exclusivamente por espécies nativas. Por último, ficou definido o estrato de regeneração natural, com 0,3 a $6,0 \mathrm{~m}$ de altura, que apresentou um grande número de plantas (48.919 ind/ha), também formada exclusivamente por espécies nativas.

Um resultado que merece destaque é a ausência absoluta de plantas de acácia-negra nos estratos intermediário, inferior e de regeneração natural, indicando que, no período depois do estabelecimento da plantação, não houve regeneração da espécie, e como as plantas remanescentes do plantio original estão em senescência, em pouco tempo não deverão ser encontrados indivíduos vivos de acácia-negra na área. Isso afasta a possibilidade da acácia-negra tornar-se invasora desse ambiente e vir a prejudicar o desenvolvimento da regeneração natural, conforme citado por Espindola et al. (2005) para a utilização de espécies exóticas na recuperação ambiental. O sub-bosque de Acacia auriculiformis A. Cunn. Ex. Benth., Acacia mangium e Mimosa tenuiflora (Willd.) Poir. também não apresentou regeneração dessas espécies (CHADA et al., 2004). Porém, o mesmo fato não tem ocorrido em plantios de Leucena e Pinus.

Plantio de Leucaena leucocephala (Lam.) de Wit. apresentou 98\% da regeneração natural composta por indivíduos desta espécie (FREIRE et al., 2000). Andrae et al. (2005) encontraram, em média, 33.000 plantas/ha de Pinus spp. no sub-bosque dessa espécie, no entanto, observaram que a distribuição entre os povoamentos avaliados foi bastante irregular. 


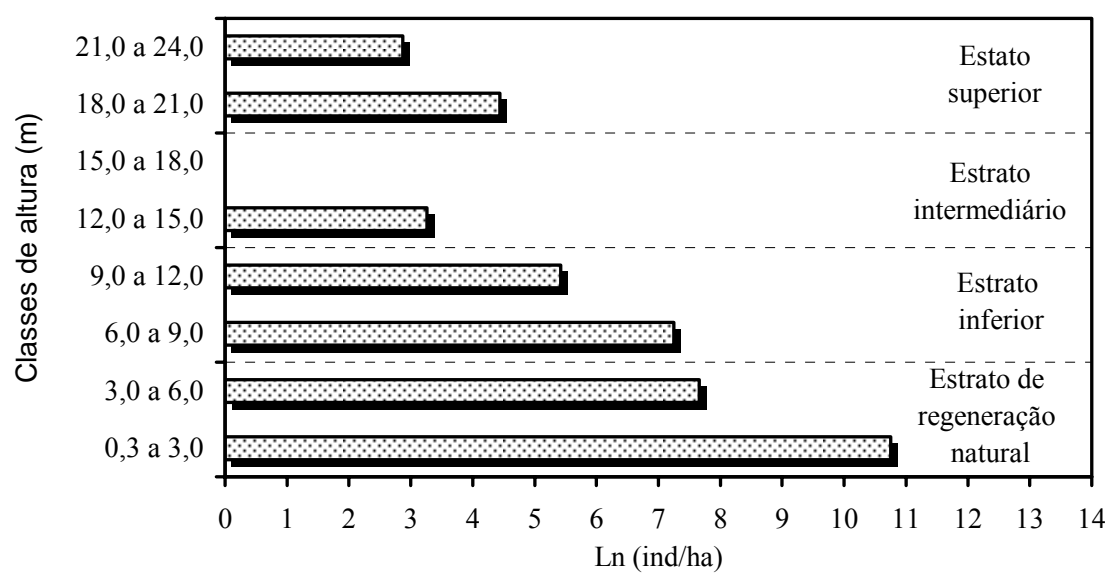

FIGURA 3: Distribuição em classes de altura e estratificação da vegetação de um povoamento de acácia-negra, em Cristal, RS.

FIGURE 3: Height class and vegetation stratification of a black-wattle plantation, in Cristal, RS, Brazil.

Esses resultados indicaram que a acácia-negra não é uma invasora de ambientes antrópicos de mata ripária da Floresta Estacional Semidecidual, desde que haja disponibilidade de propágulos para o estabelecimento de espécies nativas. Segundo Richardson et al. (2000), uma espécie deve ser considerada invasora de um determinado ambiente quando sustenta uma população durante vários ciclos de vida e que seja capaz de ocupar áreas vizinhas ao povoamento original. Neste trabalho, constatou-se que a acácia-negra foi incapaz de regenerar na área em que foi inicialmente plantada, bem como não se expandiu para os fragmentos florestais circunvizinhos. Além do mais, permitiu uma exuberante regeneração de espécies nativas em seu sub-bosque e, dessa forma, pode ter contribuído para a ocorrência de um modelo facilitador da sucessão secundária (CONNELL e SLATYER, 1977).

Entretanto, a utilização da acácia-negra para restauração de áreas degradadas apresenta a restrição da formação de um banco de sementes dessa espécie no solo, que permanecem viáveis por vários anos (PIETERSE e BOUCHER, 1997). Caso o ambiente em restauração ou áreas circunvizinhas sejam expostos a perturbações que exponha o solo à plena luz, essas sementes poderão originar uma nova população de plantas e iniciar um processo de invasão da acácia-negra (MOCHIUTTI et al., 2007).

\section{Estratégia de dispersão e de crescimento}

Das espécies amostradas, 84\% (41) possuem dispersão zoocórica, 12\% (6) anemocórica e 4\% (2) autocórica (Tabela 1). A predominância de espécies zoocóricas na regeneração natural também foi observada no sub-bosque de Pinus (LOMBARDI e MOTTA JÚNIOT, 1992) e Eucalyptus (NERI et al., 2005). Considerando o número de indivíduos, verifica-se que $95,9 \%$ das plantas da regeneração natural apresentaram dispersão zoocórica, 3,8\% anemocórica e somente $0,3 \%$ a síndrome autocórica. A dispersão de sementes por aves foi facilitada pelos poleiros naturais formados pelas plantas mortas de acácia-negra existentes na área. A predominância da dispersão zoocórica contribuiu para a alta diversidade da regeneração natural observada neste trabalho e indica que mesmo plantações localizadas mais distantes de fragmentos nativos podem apresentar uma diversificada regeneração natural em seu sub-bosque.

O grupo ecológico predominante no sub-bosque do povoamento de acácia-negra foi o das espécies clímax exigente de luz, que apresentou a maior densidade relativa no estrato arbóreo (46\%) e nas classes II (54\%) e III (69\%) do estrato de regeneração natural (Figura 4). A proporção indivíduos de espécies pioneiras foi de $43 \%$ no estrato arbóreo, reduzindo-se no estrato de regeneração natural para $14 \%$. O contrário ocorreu com as espécies clímax tolerantes a sombra, que representaram $8 \%$ dos indivíduos do estrato arbóreo e $42 \%$ da classe I do estrato de regeneração natural. A maior proporção de plantas de espécies exigentes de luz no estrato arbóreo e na classe mais alta do estrato de regeneração natural e o ingresso de indivíduos de espécies tolerantes a sombra nas classes de menores tamanho do estrato de regeneração natural indica o avanço do processo de sucessão florestal na área do povoamento de acácia-negra. 


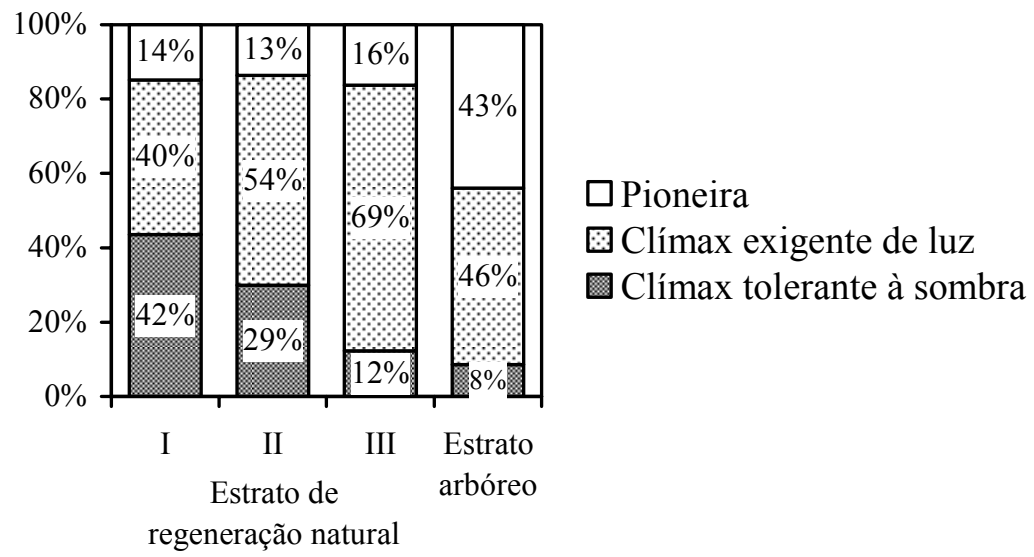

FIGURA 4: Porcentagem do número de indivíduos por grupo ecológico nos estratos arbóreo e de regeneração natural (classes de altura I, II e III) de um povoamento de acácia-negra, em Cristal, RS.

FIGURE 4: Proportion of the number individuals by ecological group in the tree and natural regeneration (I, II and III class height) stratus in a black-wattle plantation, in Cristal, RS, Brazil.

\section{CONCLUSÕES}

O povoamento abandonado de acácia-negra proporciona uma abundante e diversificada regeneração de espécies florestais nativas em seu sub-bosque, sendo capaz de restabelecer boa parte da riqueza florística original, podendo ser indicada para ativar mecanismos que facilitem a regeneração natural de áreas degradadas.

As espécies nativas que apresentam o maior valor de importância no estrato arbóreo são Casearia sylvestris, Myrsine lorentziana, Zanthoxylum petiolare, Gochnatia polimorpha, Casearia decandra, Myrsine umbellata, Ocotea puberula e Myrcia glaba. No estrato de regeneração natural, destacam-se, pela maior densidade de plantas, Faramea marginata, Myrsine lorentziana, Myrsia glabra, Casearia decandra, Daphnopsis racemosa, Casearia sylvestris, Myrsine coriacea, Myrsine umbellata, Allophylus edulis e Ocotea pulchella

A síndrome de dispersão zoocórica é a predominante entre as espécies presentes no sub-bosque do povoamento de acácia-negra, que pode ter sido responsável pela alta diversidade florística encontrada neste trabalho.

A sucessão florestal encontra-se em um processo de evolução, no qual ocorre uma maior densidade de espécies exigentes de luz nos estratos superiores e um ingresso de indivíduos tolerantes a sombra na classe inferior da regeneração.

A alta mortalidade das plantas da plantação original e a ausência absoluta da acácia-negra no sub-bosque indicam que essa espécie não se constitui numa invasora da mata ripária da Floresta Estacional Semidecidual, desde que haja disponibilidade de propágulos de espécies nativas para recolonizar a área.

\section{AGRADECIMENTOS}

Ao Sr. Arlindo Debatini, pelas informações do histórico da área e identificação do nome popular das espécies. Aos colegas Marcos Behling, Jéferson de Oliveira e Ademir Luís da Silva pelo auxilio nos trabalhos de campo e na coleta e preparação do material botânico. Aos pesquisadores J. G. Kray, J. A. Jaerenkow e J. Budke, pela contribuição na identificação taxonômica das espécies. 


\section{REFERÊNCIAS BIBLIOGRÁFICAS}

ABRAF. Anuário estatístico da ABRAF: ano base 2006. Brasília, 2007. 80 p.

ANDRAE, F.H.; PALUMBO, R.; MARCHIORI, J.N.C.; DURLO, M.A. O sub-bosque de reflorestamentos de pinus em sítios degradados na região da Floresta Estacional Decidual no Rio Grande do Sul. Ciência Florestal, Santa Maria, V. 15, n. 1, p. 43-63, 2005.

AUER, C.G.; SILVA, R. Fixação de nitrogênio em espécies arbóreas. In: CARDOSO, E.J.B.N.; TSI, M.; NEVES, M.C.P. Microbiologia do solo. Campinas: Universidade Estadual de São Paulo, 1992. p. 160-167.

BACKES, P.; IRGANG, B. Árvores do Sul: guia de identificação e interesse ecológico. Santa Cruz do Sul: Programa Clube da Árvore, Instituto Souza Cruz, 2002. 328 p.

CALEGARIO, N.; SOUZA, A.L.; MARANGON, L.C.; SILVA, A.F. Parâmetros florísticos e fitossociológicos da regeneração natural de espécies arbóreas nativas no sub-bosque de povoamentos de Eucalyptus. Revista árvore, Viçosa, v. 17, n. 1, p. 16-29. 1993

CAMPELLO, E.F.C. A influência de leguminosas arbóreas fixadoras de nitrogênio na sucessão vegetal em áreas degradadas na Amazônia. Viçosa: UFV, 1999. 121 p. Tese (Doutorado em Ciência Florestal) - Universidade Federal de Viçosa, Viçosa, 1999.

CARVALHO, P.E.R. Espécies florestais brasileiras: recomendações silviculturais, potencialidades e uso da madeira. Colombo: Embrapa CNPF, 1994. 640 p.

CARVALHO, P.E.R. Espécies arbóreas brasileiras. Colombo: Embrapa Floresta, 2003. v. 1, 1039 p.

CARVALHO, P.E.R. Espécies arbóreas brasileiras. Colombo: Embrapa Floresta, 2006. v. 2, 627 p.

CHADA, S.S.; CAMPELLO, E.F.C.; FARIA, S.M. Sucessão vegetal em uma encosta reflorestada com leguminosas arbóreas em Angra dos Reis, RJ. Revista Árvore, Viçosa, v. 28, n. 6, p. 801-809, 2004.

CONNEL, J.H.; SLATYER, R.O. Mechanisms of succession in natural communities and their role in community stability and organization. American Naturalist, v. 111, p. 1119-1144, 1977.

ESPÍNDOLA, M.B.; BECHARA, F.C.; BAZZO, M.S.; REIS, A. Recuperação ambiental e contaminação biológica: aspectos ecológicos e legais. Biotemas, Florianópolis, v. 18, n. 1, p. 27-38, 2005.

FREIRE, J.M.; PORTELA, R.; SANTANA, C.A.A.; SANTOS, C.J.; FARIA, S.M. Regeneração natural sob plantios com predominância de Leucaena leucocephala (Lam.) De Wit., Mimosa caesalpoiniifolia Benth. e plantio com maior diversidade de espécies em Madureira, RJ. In: FOREST, Porto Seguro, 2000. Anais...Porto Seguro: Biosfera, 2000. p. $181-183$

HEALEY, S.P.; GARA, R.I. The efecto of a teak (Tectona grandis) plantation on the establishment of native species in an abandoned pasture in Costa Rica. Forest Ecology and Management, v. 176, p. 497-507, 2003.

HEAR - Hawaiian Ecosystems at Risk Project. Acacia mearnsii. Disponível em $<$ http://www.hear.org/pier/species/acacia_mearnsii.htm> Acesso em 22 fev. 2005.

HENDERSON, L. The Southern African Plant Invaders Atlas (SAPIA) database and bibliography. In: MACDONALD, I.AW. et al. (Ed.). Invasive Alien Species in Southern Africa: National Reports e Directory of Resources. Cape Town: Global Invasive Species Programme, 2003. p. 91-125.

IBGE. Mapa de vegetação do Brasil. Rio de Janeiro: IBGE, 2004 Disponível em $<\mathrm{http}$ :/www.ibge.gov.br/servidor_arquivos_geo> Diretório: mapas/tematicos/mapas_murais/vegetacao.pdf Acesso em 14 Fev. 2006.

KANNEGIESSER, U. Apuntes sobre algunas acacias australianas: Acácia mearnsii De Willd. Ciência e Investigación Forestal, Santiago, v. 4, n. 2, p. 198-212, 1990.

LOMBARDI, J.A.; MOTTA JR, J.C. Levantamento do subbosque de um reflorestamento monoespecífico de Pinus elliottii em relação as síndromes de dispersão. Turrialba, v. 42, n. 4, p. 438-442, 1992.

LORENZI, H. Árvores brasileiras: manual de identificação e cultivo de plantas arbóreas nativas do Brasil. Nova Odessa: Plantarum, v. 1, 1992. 352 p.

LORENZI, H. Árvores brasileiras: manual de identificação e cultivo de plantas arbóreas nativas do Brasil. Nova Odessa: Plantarum, v. 2, 1998. 368 p.

LOWE, S.; BROWNE, M.; BOUDJELAS, S.; De POORTE, M. 100 of the World's Worst Invasive Alien Species: A selection from the Global Invasive Species Database. Auckland: ISSG/SSC/IUCN, 2004. 12 p. 
LUGO, A.E. The apparent paradox reestablishing species richness on degraded lands with tree monocultures. Forest Ecology and Management, v. 99, n. 1,2, p. 9-19, 1997.

MAGURRAN, A.E. Ecological diversity and its measurement. Princeton: Princeton University Press, 1988.179 p.

MARTINS-CORDER, M.P; BORGES, R. Z.; BORGES JUNIOR, N. Fotoperiodismo e quebra de dormência em sementes de acácia-negra (Acacia mearnsii De Wild.). Ciência Florestal, Santa Maria, v. 9, n. 1, p. 71-77, 1999.

MOCHIUTTI, S.; HIGA, A.R.; SIMON, A.A. Susceptibilidade de ambientes campestres a invasão de acácia-negra (Acacia mearnsii DE WILD.) no Rio Grande do Sul. Revista Floresta, Curitiba, v. 37, n. 2, p., 2007.

MUELLER-DOMBOIS, D.; ELLENBERG, H. Aims and methods of vegetation ecology. New York: John Willey \& Sons, $1974.547 \mathrm{p}$.

NAPPO, M.E.; OLIVEIRA FILHO, A.T.; MARTINS, S.V. A estrutura do sub-bosque de povoamentos homogêneos de Mimosa scabrella Bentham, em área minerada, em Poços de Caldas, MG. Ciência Florestal, Santa Maria, v. 10, n. 2, p. 17-29, 2000.

NAPPO, M.E.; GRIFFITH, J.J.; MARTINS, S.V.; MARCO JR, P.; SOUZA, A.L.; OLIVEIRA FILHO, A.T. Dinâmica da estrutura fitossociológica da regeneração natural em sub-bosque de Mimosa scabrella Bentham em área minerada, em Poços de Caldas, MG. Revista Árvore, Viçosa, v. 28, n. 6, p. 811-829, 2004.

NERI, A.V.; CAMPOS, E.P.; DUARTE, T.G.; MEIRA NETO, J.A.A.; SILVA, A.F.; VALENTE, G.E. Regeneração de espécies nativas lenhosas sob plantio de Eucalyptus em área de cerrado na Floresta Nacional de Paraopeba, MG, Brasil. Acta Botânica Brasílica, São Paulo, v. 19, n. 2, p. 369-376, 2005.

OLIVEIRA FILHO, A. Estudos ecológicos da vegetação como subsídio para programas de revegetação com espécies nativas: uma proposta metodológica. Cerne, Lavras, v. 1, n. 1, p. 64-72. 1994.

PARROTTA, J.A.; TURNBULL, J.W.; JONES, N. Catalyzing native forest regeneration on degraded tropical lands. Forest Ecology and Management, v. 99, n. 1,2, p. 1-7, 1997a.

PARROTTA, J.A.; KNOWLES, O.H.; WUNDERLE JR, J.M. $\quad$ Development of floristic diversity in 10-year-old restoration forests on a bauxite mined site in Amazonia. Forest Ecology and Management, v. 99, n. 1,2, p. 21-42, $1997 \mathrm{~b}$.

PIETERSE, P.J.; BOUCHER, C. Is Burning a Standing Population of Invasive Legumes a Viable Control Method? Effects of a Wildfire on an Acacia mearnsii Population. Southern African Forestry Journal, n. 180, p. 15-21. 1997.

REIS, A.; ZAMBONIN, R.M.; NAKAZONO, E.M. Recuperação de áreas florestais degradadas utilizando a sucessão e as interações planta-animal. São Paulo: Conselho Nacional da Reserva da Biosfera da Mata Atlântica, 1999. $43 \mathrm{p}$.

REITZ, R.; KLEIN, R.M.; REIS, A. Projeto Madeira do Rio Grande do Sul. Itajaí: Herbário Barbosa Rodrigues, 1988. $525 \mathrm{p}$.

RICHARDSON, D.M. et al. Naturalization and invasion of alien plants: concepts and definitions. Diversity and distribuitions, v. 6, p. 93-107. 2000.

RIO GRANDE SO SUL. Macrozoneamento agroecológico e econômico do Estado do Rio Grande do Sul. Porto Alegre: Secretaria da Agricultura e Abastecimento, 1994. 307 p.

RONDON NETO, R.M.; BOTELHO, S.A.; FONTES, M.A.L.; DAVIDE, A.C.; FARIA, J.M.R. Estrutura e composição florística da comunidade arbustivo-arbórea de uma clareira de origem antrópica, em uma Floresta Estacional Semidecidua Montana, Lavras-MG, Brasil. Cerne, Lavras, v. 6, n. 2, p. 79-94. 2000.

SAPORETTI JR., A.W.; MEIRA NETO, J.A.A.; ALMADA, R. Fitossociologia de sub-bosque de cerrado em talhão de Eucalyptus grandis W. Hill ex Maiden no Município de Bom Despacho-MG. Revista Árvore, Viçosa, v. 27, n. 6, p. 905-910, 2003.

SARTORI, M.S.; POGGIANI, F.; ENGEL, V.L. Regeneração da vegetação arbórea nativa no sub-bosque de um povoamento de Eucalyptus saligna Smith. localizado no estado de São Paulo. Scientia forestalis, Piracicaba, n. 62, p. 86-103, 2002.

SCHUMACHER, M.V.; BRUN, E.J.; RODRIGUES, L.M.; SANTOS, E.M. Retorno de nutrientes via deposição de serapilheira em um povoamento de acácia-negra (Acacia mearnsii De Wild.) no Estado do Rio Grande do Sul. Revista Árvore, Viçosa, v.27, n.6, p.791-798, 2003.

SEMA. Relatório final do inventário florestal contínuo do Rio Grande do Sul. Porto Alegre: Sema, 2001. Disponível em < http://coralx.ufsm.br/ifcrs/frame.htm > acesso em 18 Fev. 2006 
SILVA JR., M.C.; SCARANO, F.R.; CARDEL, F.S. Regeneration of an Atlantic forest formation in the understorey of a Eucalyptus grandis plantation in south-eastern Brazil. Journal of Tropical Ecology, v. 11, p. 147-152, 1995.

SHERY, S.P. The Black Wattle (Acacia mearnsii De Wild.). Pietermaritzburg: University of Natal Press, 1971. 402 p.

STEIN, P.P.; TONIETTO, L. Black Watle Silviculture in Brazil. In: BROWN, A.G.; KO, H.C. (Ed.). Black Wattle and its Utilization. Barton: RIRDC, 1997. p. 78-82.

VELOSO, H.P.; RANGEL-FILHO, A.L.R.; LIMA, J.C.A. Classificação da vegetação brasileira adaptada a um sistema universal. Rio de Janeiro; IBGE, 1991. 124 p.

WUNDERLE JR, J.M. The role of animal seed dispersal in accelerating native forest regeneration on degraded tropical lands. Forest Ecology and Management, v. 99, n. 1,2, p. 223-235, 1997. 